\title{
GLAD!
}

Revue sur le langage, le genre, les sexualités

$05 \mid 2018$

Raconter les sexualités depuis la marge

\section{« Je ne suis pas homme de lettres ni littérateur de profession $»$ : la question de l'auteur.e dans les autobiographies de courtisanes du XIX ${ }^{\mathrm{e}}$ siècle}

"I am no Man of Letters or Professional Male Writer": the Question of Authorship and Gender in XIXth Century Courtesans' Autobiographies

Lola Gonzalez-Quijano

\section{OpenEdition}

Journals

Édition électronique

URL : http://journals.openedition.org/glad/1447

ISSN : 2551-0819

Éditeur

Association GSL

Référence électronique

Lola Gonzalez-Quijano, « « Je ne suis pas homme de lettres ni littérateur de profession » : la question de l'auteur.e dans les autobiographies de courtisanes du XIX siècle », GLAD! [En ligne], 05 | 2018, mis en ligne le 15 décembre 2018, consulté le 17 décembre 2020. URL : http://journals.openedition.org/ $\mathrm{glad} / 1447$

Ce document a été généré automatiquement le 17 décembre 2020.

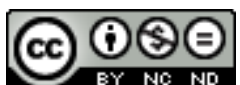

La revue GLAD! est mise à disposition selon les termes de la Licence Creative Commons Attribution Pas d'Utilisation Commerciale - Pas de Modification 4.0 International. 


\title{
" Je ne suis pas homme de lettres ni littérateur de profession » : la question de l'auteur.e dans les autobiographies de courtisanes du $\mathrm{XIX}^{\mathrm{e}}$ siècle
}

\author{
"I am no Man of Letters or Professional Male Writer" : the Question of \\ Authorship and Gender in XIXth Century Courtesans' Autobiographies
}

Lola Gonzalez-Quijano

Quand bien même reste-t-il sulfureux, le témoignage de travailleur.es du sexe, avec des auteures comme Grisélidis Réal ou Nelly Arcan, s'apparente aujourd'hui à un sousgenre littéraire qui a su acquérir ses lettres de noblesse (Larochelle 2015). Au $\mathrm{XIX}^{\mathrm{e}}$ siècle, il en va tout autrement. La prostitution féminine est au cœur d'une abondante production textuelle et visuelle: pièces de théâtre, tableaux, caricatures, sculptures, romans, mais également essais, ouvrages juridiques ou traités médicaux évoquent les heurs et malheurs des femmes qui "vendent » leur corps. Une grande partie de cette production est désormais tombée dans l'oubli mais la Nana d'Emile Zola, l'Olympia de Manet, la Fille Elisa d'Edmond de Goncourt ou encore la Mélita de l'opérabouffe La Vie parisienne continuent d'incarner dans l'imaginaire collectif les prostituées de cette époque. Pourtant, ces représentations sont presque exclusivement élaborées par des hommes (Dottin-Orsini \& Grojnowski 2008) et sont davantage des créations discursives que des peintures fidèles du monde de la prostitution (Bernheimer 1989, Clayson 1991, Matlock 1994).

Dans ce contexte, et bien que le discours masculin sur la prostitution soit loin d'être uniforme, les autobiographies de femmes galantes et d'actrices - les deux catégories se confondent largement à l'époque -, qui se multiplient dans la seconde moitié du XIX ${ }^{e}$ siècle, ont été perçues comme l'émergence d'une voix féminine et singulière. Pour la première fois en effet, des prostituées prennent la plume "pour s'exprimer et 
s'autoreprésenter " (Koliopanos 2015: 104) et surtout le font pour répondre aux représentations masculines de la prostituée (Sullivan 2016). Selon Courtney Sullivan, les ouvrages que Liane de Pougy publie à la Belle Epoque seraient même plus réalistes que les fictions de la prostitution dont les auteurs sont des hommes car attachés à décrire fidèlement une réalité connue (Sullivan 2016). Cette affirmation est cependant difficile à défendre à partir de leur seule analyse textuelle tant les Mémoires de courtisanes se caractérisent par leur tendance à l'hagiographie et leur volonté de créer un mythe (Jouanny 2002), qui induit de fait un effacement des pratiques prostitutionnelles (Koliopanos 2015). Les autobiographies d'actrices et de courtisanes sont soumises à une tension permanente entre besoin de répondre aux goûts licencieux du public et envie de paraitre sous son meilleur jour, nécessité de défendre son honorabilité féminine et souci de mettre en scène une trajectoire de femme exceptionnelle à rebours des destins féminins traditionnels. Dans bien des cas, la publication de Mémoires semble ainsi avoir été la touche finale, la dernière pierre à l'édification d'un personnage de femme fatale, séductrice et talentueuse savamment créé tout au long d'une vie (Gonzalez-Quijano 2015b).

Par ailleurs, ces ouvrages font l'objet, au moment de leur parution, non seulement d'opérations de lancement dans les journaux mais aussi de vives polémiques quant à leur moralité et leur véritable auteur, qui les insèrent pleinement dans les enjeux littéraires de leur temps. Et ce d'autant plus que ces publications sont indubitablement des succès de librairies : 40000 exemplaires des Mémoires de Thérésa (1865) sont vendus en huit jours, les Souvenirs (1926) de la Belle Otéro sont réédités 22 fois l'année de leur parution, Ma Double vie (1907) de Sarah Bernhardt fait l'objet de traductions en allemand, anglais et italien dans les deux années qui suivent sa parution. Afin de prendre en compte cette situation éditoriale somme toute particulière et de dépasser les oppositions quelque peu schématiques entre fiction et autobiographie, écriture masculine et féminine sur la prostitution, il faut analyser très concrètement le contexte de production du sous-genre de l'autobiographie de courtisane au XIX siècle. À partir d'un corpus de 26 ouvrages $^{1}$ et de recherches prosopographiques, il s'agit d'interroger le lien entre écriture et récit autobiographique en se demandant si les courtisanes sont les véritables auteures de leurs œuvres. Non pas pour décrédibiliser leurs velléités littéraires et ricaner sur l'impossibilité pour une femme d'être auteur, à l'instar de nombreux journalistes et hommes de lettres à la parution de leurs œuvres (Lorusso 2017), mais pour entrevoir comment l'aide auctoriale qu'elles ont reçue a pu influencer «l'écriture » et le récit de la prostitution.

\section{De l'auteur.e et de l'héroïne dans les mémoires de courtisanes}

Le terme d'autrice recule progressivement dans les usages à partir du XVII ${ }^{\mathrm{e}}$ siècle et, au $\mathrm{XIX}^{\mathrm{e}}$ siècle, les femmes écrivains ne sauraient être que des "bas-bleus ${ }^{2}$ " aigris et rabougris enviant le génie masculin (Planté 1989). Dans un siècle qui fait de la beauté et de l'intelligence deux catégories antinomiques pour les femmes, les courtisanes qui publient, sont particulièrement suspectées de ne pas avoir écrit la moindre ligne de leur œuvre, et ce d'autant plus que le récit de la prostitution est alors un genre littéraire exclusivement masculin (Reid 2011). Mais qu'en est-il réellement? 


\section{L'auteur.e en tant que construction éditoriale}

5 Dans le prologue des Mémoires de Rose Pompon', leur auteur déclaré, Louis de Vallières, précise n'avoir jamais eu de relations personnelles avec cette dernière. Mais habitant le même immeuble, elle lui aurait légué, peu de temps avant sa mort, des notes afin qu'en tant qu'homme de lettres il s'en serve pour reconstituer sa vie. En réalité, si les Mémoires de Rose Pompon paraissent en 1877, Valérie Hamelin de son vrai nom ne meurt qu'en $1895 . .$. Ce procédé de légitimation d'une œuvre écrite sans connaitre Rose Pompon et, compte tenu de sa charge contre celle-ci, probablement sans son consentement, est révélateur des différents procédés scripturaux et éditoriaux mis en place au XIX ${ }^{\mathrm{e}}$ siècle pour distinguer les «autobiographies» de courtisanes des autres productions littéraires et journalistiques les mettant en scène ou, au contraire, pour en brouiller les frontières.

6 Telle qu'elle émerge dans la seconde moitié du XIXe siècle, l'autobiographie de courtisane doit en effet se positionner vis-à-vis de deux productions romanesques majeures mettant en scène la vie des prostituées: le "roman-mémoire » qui lui est antérieur et le "roman de filles» des littératures réaliste et naturaliste qui lui est concomitant. Au XVIIIe et dans la première moitié du XIXe siècle, les « récits de vie » de prostituées sont des ouvrages à caractère licencieux, voire pornographique, à l'instar des Mémoires d'une célèbre courtisane des environs du Palais-Royal ${ }^{4}$ ou La Belle Cauchoise ou Mémoires d'une jolie normande devenue courtisane célèbre ${ }^{5}$ (Van Crugten-André 1997). Dans ces romans-mémoires, la courtisane est à la fois personnage inventé et narratrice inventant son histoire. Elle incarne une héroïne à la lisière de la prostitution érudite comme les hétaïres antiques, en quête d'élévation sociale et d'enrichissement comme les aventurières ou les parvenues, fréquentant les hommes les plus nobles et les plus puissants comme les favorites, et talentueuse comme les actrices du théâtre ou de l'Opéra (Cortey 2001). De la Monarchie de Juillet à la Belle Époque, les écrivains français produisent une abondante série de figures de prostituées, et de livres souvent éponymes, dont certaines deviennent de véritables modèles ou archétypes comme La Dame aux Camélias (1848), La Fille Élisa (1877), Boule-de-Suif (1879) ou encore Nana (1880) (Braund 1947, Benhamou 1996, Dottin-Orsini \& Grojnowski 2008). À cette littérature romanesque il faut ajouter le sous-genre littéraire que peut constituer la littérature panoramique galante de la Monarchie de Juillet et du Second Empire avec son cortège de publications dédiées aux femmes qui s'illustrent dans les bals et les théâtres parisiens : Les Oiseaux de nuit et les polkeuses des scènes publiques ${ }^{6}$, Voyage autour de Pomaré, reine de Mabille ${ }^{7}$, Les Hétaïres en $1860^{8}$; ces écrits intégrant souvent une suite de portraits de ces dames.

7 Cette situation éditoriale explique que les ouvrages qui composent ce corpus cherchent tous à faire preuve d'authenticité. Les titres des souvenirs de Lola Montez, de Polaire et de Thérésa (Illustration $\mathrm{n}^{\circ} 1$ ) précisent qu'ils ont été écrits "par elle-même ». La plupart, dont par exemple les Mémoires de Rigolboche, d'une biche anglaise, de Thérésa, d'Eugénie Buffet, de Sarah Bernhardt et de Cléo de Mérode s'ornent d'un portrait de l'auteure (Illustration ${ }^{\circ} 2$ ). D'autres, dont ceux de Lola Montez, de Liane de Pougy et de Thérésa, affichent également un fac-similé de l'écriture de leur auteure. Ces gages d'authenticité, visibles dès la couverture et les premières pages du livre, personnalisent le livre et donnent « une incarnation » à leur auteure. Et ce d'autant plus que ces dames sont avant tout connues, et reconnues, pour leur physique. Leur image, en raison de 
leur statut de personnage publique, est alors largement véhiculée par la presse et la photographie (Corvisier 2007, Lilti 2014). Ces procédés permettent aussi très concrètement de distinguer, dans les librairies ou les cabinets de lecture, ces récits autobiographiques des "portraits d'actrices" et des biographies écrites par des journalistes en vogue avec lesquels ils sont en concurrence. Cependant, pour un œil averti, cette authenticité tourne vite à la farce, comme pour les Mémoires de Finette dont la couverture est agrémentée d'une épigraphe de la prétendue auteure : «[...] je ne suis pas homme de lettres ni littérateur de profession. En voici la preuve. »

\section{Illettrisme et fausses autobiographies}

Les auteures étudiées dans cet article ont toutes appartenu, à des degrés divers et de façon plus ou moins longue, à ce que l'on appelle au XIX siècle le "demi-monde ». L'expression, inventée par Alexandre Dumas fils en $1854^{9}$ remporte un très vif succès et, très vite, sert à qualifier l'ensemble des femmes entretenues, des courtisanes et des actrices dans la mouvance de la vie parisienne. Contrairement aux prostituées de rue et autres filles publiques, ces femmes n'ont guère à craindre de la police, même lorsqu'elles sont étroitement surveillées par celle-ci (Berlière 1992); et si elles sont stigmatisées par leur activité prostitutionnelle, c'est aussi celle-ci qui leur vaut, paradoxalement, succès, célébrité et fortune (Gonzalez-Quijano 2012). Tirant son modèle des salons de l'aristocratie (Lilti 2005), le demi-monde est un espace où les femmes se doivent de briller, certes par leur beauté, mais aussi par leur exubérance et leur personnalité, leur art de la conversation et du jeu de mots. Mais passer de l'oral à l'écrit n'est pas chose aisée. Et ce d'autant plus que, derrière leur commune célébrité, ces femmes cachent des trajectoires individuelles et des parcours de mobilité sociale extrêmement divers qui impactent directement leur rapport à l'écrit. La majorité des demi-mondaines sont issues des couches populaires et ont un passé d'ouvrière, de domestique, de demoiselle de magasin, de prostituée occasionnelle ou de fille publique (Gonzalez-Quijano 2012). Fille d'une marchande de poissons, Camille Ackinson est, par exemple, connue dans le monde galant sous le surnom de «tige de bottes». Surnom inventé par le journaliste et homme de lettres Edmond Tarbé des Sablons qui l'aurait baptisée ainsi à cause des nombreux «cuirs ${ }^{10}$ " qu'elle fait dans la conversation. Le policier qui rédige sa fiche en 1872 n'hésite pas d'ailleurs à la décrire comme « des plus communes, sans instruction et sans éducation aucune ${ }^{11}$ ».

Cette absence d'instruction des demi-mondaines fait sens par rapport à l'histoire de l'éducation au XIX siècle (Giolitto 1983-84). Elle explique également qu'un certain nombre d'autobiographies de courtisanes publiées à cette époque soient des faux. Il y a tout d'abord des biographies fictionnalisées qui jouent de l'ambiguïté entre titre et auteur, comme les Mémoires de Rose Pompon publiés sous le nom de Louis de Vallières, ou qui modifient directement le nom de l'auteur, comme pour les Mémoires d'une biche anglaise $^{12}$ attribués à "Quillette", du surnom Skittles de la courtisane Catherine Walters, et qui ne sont en réalité que la traduction d'une biographie anglaise ${ }^{13}$. Ensuite viennent de véritables fausses autobiographies, c'est le cas notamment des Mémoires de Rigolboch ${ }^{14}$, désormais attribués à Ernest Blum \& Louis Huart, des Mémoires de Thérésa par elle-même $e^{15}$, rédigés par Ernest Blum, Henri Rochefort \& Albert Wolff, et des Mémoires de Finette bien qu'il n'ait pas été possible d'en identifier le véritable auteur. Même chose pour Lola Montès. Aventures de la célèbre danseuse racontées par elle-même ${ }^{16}$ qui a certainement été écrit par Hugues Gabriel Marie, comte de Coral, avocat et hommes 
de lettres (Wagneur \& Cesteur 2012). C'est d'ailleurs sous l'un de ses pseudonymes, Hugues de Cortal, que parait leur traduction en allemand ${ }^{17}$.

Quelques auteures assument avoir collaboré avec des hommes de lettres à l'instar de Polaire qui, lors de la parution de Polaire par elle-même, déclare qu'elle a demandé à son "ami Charles Cluny, qui a déjà rendu le même service à Mayol, de mettre mon manuscrit en ordre, et en bon français [...] ${ }^{18}$ »Certains procédés scripturaux semblent s'apparenter à ce qui se fait aujourd'hui en termes d'autobiographie sous la forme d'entretiens avec un journaliste pour certaines personnalités issues du monde sportif ou artistique. Le titre des Mémoires d'Eugénie Buffet, Ma vie, mes amours, mes aventures, est ainsi accompagné d'une mention précisant qu'il s'agit de "confidences recueillies par Maurice Hamel ${ }^{19}$ ", idem pour les Mémoires de Casque d'or racontés par elle-même et publiés par Henri Frémont ${ }^{20}$. Publiés au $\mathrm{XX}^{\mathrm{e}}$ siècle, et par rapport aux fausses autobiographies évoquées précédemment, ces ouvrages sont avant tout révélateurs du renforcement du cadre juridique encadrant droit d'auteur et travail de prête-plume, entre les deux siècles (Edelman 2004). Les Souvenirs de la Belle Otéro font ainsi apparaitre le seul nom de Caroline Otéro sur la couverture mais la $2^{\mathrm{e}}$ page indique «présentés et préfacés par Claude Valmont », bien qu'il n'y ait pas la moindre préface ni présentation, tandis que la $3^{\mathrm{e}}$ page précise « copyright by Caroline Otéro et Claude Valmont $1926 »^{21}$.

11 Toutes ces femmes, à l'exception de Lola Montez, ont en commun d'être issues d'un milieu populaire voire très populaire. Les parents de Rigolboche et de Thérésa sont tailleurs d'habits, le père de Rose Pompon ferblantier, la mère de la Belle Otéro - elle n'a pas été reconnue par son père - est danseuse de rue, diseuse de bonne aventure et certainement prostituée occasionnelle. Mais surtout toutes ces femmes sont arrivées à la gloire par la danse ou la chanson. Activités artistiques où la maitrise de l'écrit n'est pas nécessaire. Cette situation laisse ces femmes à la merci de leur « autobiographe » et de ses talents de conteur au moment où il s'agit de raconter leur vie. Ce qui ne veut pas dire qu'il n'y a pas d'entente ou accord sur ce qui peut/doit être écrit. Vingt-cinq ans après la parution des Mémoires de Thérésa, Albert Wolff raconte ainsi qu'il avait inventé un père musicien et une mère qui économisait sur le diner pour faire donner de l'éducation à sa fille :

« Ni vus, ni connus, cette paire de parents, ce qui n'empêchait pas Thérésa de pleurer à chaudes larmes quand je lui ai lu le récit de son enfance. Aujourd'hui encore, elle en parle avec émotion, car elle a fini par croire que c'était arrivé $^{22}$."

Lorsqu'il y a réel dialogue entre l'auteure officielle et son autobiographe, le processus d'écriture d'une autobiographie de courtisane peut s'apparenter, par bien des aspects, à la recherche documentaire nécessaire à l'écriture d'un « roman de filles ». Afin d'écrire La Fille Elisa, les frères Goncourt accumulent des documents de toutes sortes, lisent certaines œuvres du XVIII ${ }^{\mathrm{e}}$ siècle et recueillent surtout les confidences de Maria, leur maitresse commune ${ }^{23}$ (Ricatte 1960). Pour Nana, Émile Zola fait parler ses amis intimes mais rencontre également Valtesse de la Bigne ${ }^{24}$. Ce qui laisse ces messieurs à la merci de ce que veulent bien leur raconter les demi-mondaines de leur passé et de leur ascension sociale et explique sans doute que, dans les autobiographies de courtisanes, le récit de certaines étapes, et notamment l'entrée dans la prostitution, soient équivoques, pour ne pas dire totalement invraisemblables (Gonzales-Quijano 2015). Céleste Mogador rapporte ainsi qu'après avoir fui les attouchements de son «beaupère ", elle fut recueillie par une prostituée au grand cœur. Arrêtée par erreur en 
compagnie de celle-ci, elle passe quelques temps à la prison hôpital de Saint-Lazare pour prostitution clandestine. Ce qui relève de la tragique erreur puisque, selon elle, ce n'est qu'à sa sortie de Saint-Lazare qu'elle entre vierge dans un bordel de luxe (et ce sans savoir réellement ce qu'elle aura à y faire ${ }^{25}$... « Le mensonge blanchit les dents ", aurait dit Marie Duplessis, l'inspiratrice de la Dame aux Camélias pour justifier sa tendance à transformer même les faits les plus facilement vérifiables.

\section{De la verve et de l'orthographe : l'écriture sous assistance}

Dehors de ces fausses autobiographies, les écrits autobiographiques consultés se situent dans un continuum d'écriture qui semble aller de la relecture orthographique et grammaticale - à laquelle n'échappe aucun auteur - à l'écriture à plusieurs mains. Il est important à cet égard de distinguer les demi-mondaines qui n'ont publié que leurs Mémoires de celles qui ont plusieurs ouvrages à leur actif et se sont illustrées dans plusieurs genres littéraires comme Yvette Guilbert, Marie Colombier, Liane de Pougy, Céleste Mogador et Sarah Bernhardt. Celles-ci ont toutes eu, bien qu'avec des succès très divers, des carrières de comédienne ; métier de la scène qui nécessite davantage de maitriser l'écrit que la danse ou la chanson (Martin-Fugier 2001). En ce qui concerne Yvette Guilbert, elle n'est pas uniquement chanteuse mais également auteure, compositrice, et arrangeuse de textes (anciens) et de musique ${ }^{26}$. Aujourd'hui reconnue comme artiste à part entière, Yvette Guilbert a en effet connu à ses débuts une trajectoire classique dans le demi-monde : passant d'ouvrière à demoiselle de magasin, puis de femme galante à femme entretenue, elle cherche par la suite à obtenir un statut plus légitime et des revenus plus indépendants en s'essayant d'abord au théâtre puis en rencontrant le succès dans la chanson.

Mais la maitrise de l'écrit n'implique pas d'avoir du style ou une écriture littéraire. Alors qu'au XIXe siècle, on forme essentiellement les jeunes filles à être de bonnes et pieuses ménagères (Mayeur 1979, Dinet 2011), les différences non seulement sociales mais également sexuées expliquent la nécessité pour certaines courtisanes de recourir à des compétences littéraires masculines pour pouvoir publier leurs œuvres. Les Mémoires de Marie Colombier et de Céleste Mogador, et plus généralement leurs œuvres, posent ainsi avec acuité la question de l'articulation entre écriture et auteur.e. Toutes deux filles de mère célibataire s'entendant très mal avec leurs "beaux-pères ", elles sont mises tôt en apprentissage et quittent tout aussi vite le foyer familial. Elles vont parfaire leur instruction auprès de leurs amants du même âge mais à l'éducation plus poussée : la première en étant la concubine du futur violoniste et compositeur Charles de Bériot, la seconde auprès des étudiants du Quartier Latin, puis par la suite, auprès de son amant et futur mari, le comte Lionel de Chabrillan. Selon Auriant, Marie Colombier avait une orthographe fantaisiste et $"$ manquait de toute espèce de style ${ }^{27}$. Françoise Moser, première biographe de Céleste Mogador, a eu la chance de retrouver les mémoires inédits de celle-ci sous la forme d'une quinzaine de cahiers d'écoliers couverts d'une écriture qu'elle décrit comme «si personnelle, si énergique, si dédaigneuse de la bonne volonté du lecteur, si dédaigneuse aussi de l'orthographe, et qui révèle à la fois, dans son allure fougueuse et dans son désordre, la très grande dame et la fille» (Moser $1935: 7$ ).

Céleste Mogador est l'auteure déclarée d'au moins 34 œuvres romanesques et théâtrales. Mais il est avéré aujourd'hui que son plus grand succès théâtral est une 
adaptation de son roman Les Voleurs d'or faite par Alexandre Dumas (Dumas 2006). En ce qui concerne Marie Colombier, la paternité de ses œuvres fait l'objet d'intenses discussions dans les colonnes du Mercure de France qui éclairent d'une toute autre lumière la genèse de ses livres et plus généralement les rapports qui peuvent se nouer entre courtisanes et hommes de lettres. Le Voyage de Sarah Bernhardt en Amérique et ses Mémoires sont ainsi attribués à une collaboration avec Jehan Soudan, Les Mémoires de Sarah Barnum à Paul Bonnetain et son roman On en meurt à Paul Adam. Selon l'éditeur Paul-Victor Stock, qui fut l'un des familiers de la comédienne, il lui est arrivé plusieurs fois lors de déjeuners « d'entendre l'écrivain qu'était Bonnetain donner son avis d'une façon aussi désintéressée que je le faisais moi-même, dans les discussions entre les deux collaborateurs - Marie Colombier et Jehan Soudan - sur leurs travaux passés ou en cours $^{28}$.» Autre courtisane femme de lettres, Liane de Pougy demande dans Mes cahiers bleus, écrits au jour le jour comme un journal, qu'à part les fautes grossières, "on publie le reste tel quel, par orgueil autant que par humilité, pour que cela reste bien imprégné de mon "moi"29.". Cette position peut s'expliquer par le fait que, contrairement à beaucoup de ses consœurs du demi-monde, Liane de Pougy est issue d'une famille d'officiers militaires; adolescente, elle a reçu l'éducation propre aux jeunes filles de la «bonne bourgeoisie » au couvent Saint-Anne-d'Auray (Rogers 2007). Dans le même paragraphe, cependant, Liane reconnait avoir eu recours à un correcteur, en l'occurrence l'auteur dramatique Max Maurey, pour son roman Yvée Lester.

Pour Courtney Sullivan, les hommes de lettres qui publient de faux mémoires de courtisanes, à l'instar d'Ernest Blum et de Louis Huart les auteurs des Mémoires de Rigolboche, envient le succès (littéraire) des demi-mondaines et s'approprient leur nom afin de gagner de l'argent tout en jetant le trouble sur le statut d'auteur.e des courtisanes. Hypothèse séduisante mais qui n'imagine pas que Rigolboche ait pu donner son accord pour la publication de ses Mémoires hautement fantaisistes que ce soit pour en tirer des revenus ou pour accroitre son succès médiatique. Pourtant, d'autres ouvrages de demi-mondaines, comme les Anecdotes of Love ${ }^{30}$, The Art of beauty ${ }^{31}$ de Lola Montez et les Secrets de beauté pour être belle ${ }^{32}$ d'Emilienne d'Alençon, publiés alors que leurs auteures déclarées connaissent de très grandes difficultés matérielles peuvent laisser penser qu'elles ont endossé, dans un but purement commercial et financier, la paternité d'œuvres qu'elles n'ont peut-être même pas lues. L'amour et la beauté étant les « domaines d'expertises » des courtisanes, il y a en effet un indéniable intérêt éditorial/commercial à faire apparaitre son auteur comme une demi-mondaine. Dans sa préface, d'Anecdotes of love, somme de plus de 300 pages qui évoque aussi bien les amours de Caligula que ceux du cardinal de Mazarin ou du Duc de York, Lola Montez déclare ainsi : «In writing this volume, I have consulted numerous works of historical credibility, my design being to use only such anecdotes as have the stamp of truth ${ }^{33}$ ». Travail littéraire et historique qui semble assez incompatible avec ce que l'on sait de son éducation et des dernières années de sa vie (Seymour 1998). En l'absence des manuscrits, il est cependant impossible de trancher quant à l'implication respective de l'auteure et de son "porte-plume». Force est de constater néanmoins que plus le succès est grand et le collaborateur éventuel connu, plus les défiances se font grandes vis-à-vis des auteures du corpus et de leur capacité à écrire. Suspicion que l'on pourrait être amenée à lire aujourd'hui uniquement à travers le prisme de la misogynie mais qui reflète avant tout le jeu entre «l'auteur» et la "fonction auteur» (Couturier 1995) ainsi que l'importance de la «collaboration littéraire » au XIX siècle (Stillinger 1991, Peeters 2017). 


\section{L'autobiographie galante, une collaboration littéraire} qu'à titre posthume, toutes les premières éditions des Mémoires du corpus ont été publiées du vivant de leurs auteures et, surtout, pensées dès le départ en tant que publication, c'est-à-dire en tant qu'objet commercial. Et c'est à l'aune de cette raison commerciale qu'il semble judicieux de lire la parution de ces Mémoires mais également les collaborations littéraires qui se nouent entre demi-mondaines et hommes de lettres. En effet, ces écrits sont avant tout publiés parce que les auteures, mais peut-être surtout les éditeurs, y ont vu un moyen de se faire de l'argent. Les Mémoires de Cora Pearl, Marguerite Bellanger et Fanny Lear font ainsi l'objet de rééditions à titre posthume qui ne doivent rien à la volonté de leurs auteures. Tandis que Céleste Mogador ne trouve aucun éditeur pour sa troisième série de souvenirs sans doute car, alors oubliée du public, l'opération n'est pas considérée comme rentable (Chabrillan 2015).

\section{Entre besoin d'argent et envie de se raconter}

À la parution en 1899 de Myrrhille, roman parisien autobiographique de Liane de Pougy, l'écrivaine Rachilde s'interroge: "Je n'arrive pas à comprendre, surtout devant le portrait de l'auteur, pourquoi une très puissante et une très belle femme d'amour peut désirer devenir une médiocre femme de lettre ${ }^{34}$. » La réponse est peut-être donnée par Jules Clarétie à propos de la parution d'Isola, roman autobiographique que Valtesse de la Bigne publie sous son nom de guerre « $\varepsilon \gamma \omega$ » [ego] en 1876 : «pour se confesser un peu, pour livrer ses intimes pensées, dans cette ardeur de confidences qu'elles ont toutes, avec le besoin de faire comprendre qu'elles ne ressemblent pas aux autres ${ }^{35}$ ". Albert Wolff, à propos des Mémoires de Cora Pearl, suggère, quant à lui, des raisons beaucoup plus prosaïques :

\footnotetext{
«Je ne voudrais pas offenser une femme qui, sur le tard, et par des nécessités en dehors de ses prévisions, est forcée de se jeter dans la littérature [...] C'est le dernier cri d'une belle âme qui ne veut pas disparaitre sans avoir distribué une dernière fois ses prospectus. Disons donc un éternel adieu à cette pauvre fille qui s'accroche avec un si cuisant désespoir à ce qu'elle pense être sa renommée d'hier ${ }^{36}$.»
}

Pour Carol Mossman, Céleste Mogador aurait écrit par vengeance envers sa future belle-famille et plus largement contre une société misogyne qu'elle tient pour responsable de la chute des femmes dans la prostitution et de leur stigmatisation (Mossman 2009). Mais il semblerait qu'elle y ait été incitée et aidée par son avocat, et amant d'alors, Etienne Desmarest, afin d'expliquer sa vie et sa situation au moment où, en procès, elle doit prouver qu'elle n'est pas la prostituée que l'on croit (Moser 1935). Ces femmes relatent, justifient, expliquent en effet dans leur livre une vie qui a déjà été largement commentée, et surtout par des hommes. Dans sa préface d'un Roman d'une Américaine en Russie, Fanny Lear expose :

«Mon intention est simplement de rétablir des faits dénaturés par des nouvellistes malveillants ou mal renseignés. Devenue, sans qu'il y ait eu intention ni faute de ma part, le point de mire de ces messieurs qui m'ont 
produite aux yeux de leurs lecteurs sous le jour le plus faux, - situation peu agréable à toute personne de mon sexe -, j'ai pensé qu'il serait tout aussi à propos de me mettre en pleine lumière, en ayant soin toutefois d'observer la décence et d'épargner le moindre désagrément au jeune homme qui fait l'objet de ce livre ${ }^{37}$.»

20 Cependant, ce livre est aussi une opération commerciale offrant un portrait très flatteur de l'auteure, au détriment d'ailleurs dudit jeune homme, et qui n'hésite pas à dévoiler la correspondance intime de ce dernier. Le Roman d'une Américaine en Russie sort seulement un an après l'expulsion retentissante de Fanny Lear de Russie à la suite d'un vol de diamants commis en sa faveur par son amant le grand-duc Nicolas Constantinovitch. Ce volume, par ailleurs imprimé en Belgique pour éviter d'éventuelles poursuites, a aussi pour but de lancer sa carrière en France et de "protéger » son auteure. Après deux cambriolages ratés, ayant vraisemblablement été commis par des agents de la Russie, " on » lui offre 250000 francs pour récupérer la correspondance compromettante du grand-duc mais elle tente d'obtenir le double. Sa demande ayant été refusée, elle se décide à écrire sa version de l'histoire et la faire traduire en français ${ }^{38}$; publication qui, sur le plan commercial, fut une incontestable réussite, le prix du volume ayant pu monter jusqu'à 300 francs $^{39}$.

Besoin d'argent et envie de se raconter ne sont pas incompatibles et semblent avoir été déterminants dans l'aboutissement des processus d'écriture (Iuso 1999, Cosnier 2001) et de publication. Mais Thérésa a 28 ans lorsque paraissent ses Mémoires, Rigolboche seulement 18 ! Tandis que les Confessions de Marguerite Bellanger sont publiés quatre ans avant son décès à l'âge de 48 ans et les Mémoires de Cora Pearl paraissent l'année de sa mort (elle a alors 51 ans). Il est donc nécessaire de distinguer les ouvrages publiés au moment où leurs auteures sont en pleine gloire comme les Mémoires de Rigolboche, ceux de Thérésa et de Casque d'Or, Isola, le Roman d'une Américaine en Russie ou encore les romans à clefs de Liane de Pougy ; et les mémoires et autres livres de souvenirs qui ont été écrits sur le tard, alors que la carrière artistique et galante des auteures est déclinante ou qu'elles connaissent des revers de fortune. Les premiers sont un moyen de gagner de l'argent mais surtout d'accroitre la célébrité de leur auteur.e et, par ricochet, le prix de leurs prestations artistiques et/ou vénales tandis que les seconds tentent de tirer des revenus de leur gloire d'antan.

Dans son chapitre introductif « Pourquoi j'ai fait ce livre », Cora Pearl reconnait qu'elle attend « la publication de ce volume pour avoir quelques billets de banque et essayer de $v^{v i v r e}{ }^{40}$. " Selon Albert Wolff, aucune révélation à attendre de ce livre car son auteure aurait rendu tous les papiers compromettants en sa possession moyennant des indemnités financières ${ }^{41}$. Affirmation ô combien invérifiable mais qui vient renforcer l'hypothèse selon laquelle la publication de Mémoires s'insère dans une recherche plus vaste de moyens d'existence. Il est difficile en effet pour des femmes qui ont gagné leur vie en "vendant leur corps", que ce soit de façon galante ou artistique, de trouver d'autres moyens de subsistance une fois la vieillesse venue. Valtesse de la Bigne et Cora Pearl mettent ainsi en vente une grande partie de leur mobilier $^{42}$; le catalogue faisant suite à la succession de cette dernière montre, à cet égard, combien elle vit modestement dans les dernières années de sa vie même si elle n'est pas dans un dénuement complet au moment de sa mort ${ }^{43}$. L'année où elle publie On en meurt, Marie Colombier est assignée par plusieurs de ses créanciers : la somme produite par la vente de son mobilier, 7758 francs, est attribuée à la Société des terrains du Parc Monceau à 
laquelle elle devait environ 11300 francs de loyer ${ }^{44}$. Dans les années 1890, elle publie ses Mémoires en trois volumes mais utilise, en parallèle, les mêmes stratégies que d'autres auteurs en mal d'argent comme la comtesse Dash (Mollier 2006), c'est-à-dire qu'elle réédite certaines de ses œuvres (celles qui se sont le mieux vendues) sous d'autres titres : Courte et bonne paru en 1888 reparait sous le titre de Sacha en 1898.

\section{Du secrétariat au travail de prête-plume}

Le désir de donner une autre image d'elle-même motive certainement les auteures à narrer leur vie mais le besoin d'argent est sans doute au cœur des collaborations littéraires qu'elles nouent. Le Petit Bottin des arts et des lettres, dictionnaire onomastique $\mathrm{du}$ Tout-Paris littéraire et artistique décrit ainsi Marie Colombier: "Auteur dramatique. Romancière à secrétaires. La Récamier de l'opportunisme. ». Si l'ouvrage parait de façon anonyme en 1886, l'on sait désormais qu'il est l'œuvre d'un quatuor formé par Félix Fénéon, Oscar Méténier, Jean Moréas et Paul Adam. Or, à cette époque, Paul Adam est le secrétaire de Marie Colombier ${ }^{45}$. Fils de famille ruiné, au tout début de sa carrière littéraire, il aurait été embauché par Marie Colombier sur la recommandation de Paul Alexis et c'est certainement en sa qualité de secrétaire qu'il collabore avec cette dernière sur son roman On en meurt. Dans la préface de ce dernier, adressée à Colombine [Henri Fouquier journaliste au Gil Blas], Marie Colombier ne nie pas d'ailleurs avoir eu recours à une aide littéraire sous la forme justement d'un « secrétaire »:

«Vous êtes trop fine, ô Colombine, pour faire de bourgeoises gorges chaudes devant mon oubli de l'orthographe d'Académie [...] Me garderez-vous rancune sur la question de secrétaire? Je n'en crois rien, ma chère Colombine. Les cas sont trop nombreux parmi les plus illustres de la famille. Rachel avait Crémieux. A-t-on jamais pris les poulets de Phèdre pour des plaidoiries d'avocat ? ${ }^{46}$ ».

En se rangeant derrière la grande tragédienne dont la jeunesse de misère et de mendicité était alors connue de tous, Marie Colombier se justifie en faisant appel à un précédent illustre mais dévoile plus largement l'importance du secrétaire dans l'économie scripturale du demi-monde. En effet, contrairement aux femmes, les hommes qui fréquentent le demi-monde sont issus de la noblesse française et européenne, de la finance et de la grande industrie et des élites du monde du théâtre et de la presse (Gonzalez-Quijano 2012). La correspondance amoureuse mais également mondaine (invitations, prises de rendez-vous, sollicitations, etc.) occupe une place centrale dans les relations demi-mondaines, tout en prenant un temps considérable. La gestion du courrier se retrouve ainsi souvent déléguée à des domestiques ayant plus d'instruction que leur maitresse, et qui occupent souvent une position d'« intendante " ou de «gouvernante» de la maison. Il n'est pas impossible que certaines femmes galantes aient $\mathrm{pu}$ avoir recours au service d'hommes de lettres pour leur correspondance, notamment lorsqu'il s'agit de lettres publiées dans la presse, bien que le poste de secrétaire ne devait rien avoir d'officiel. Si le journaliste Auriant assure que la relation entre Paul Adam et Marie Colombier fut toute platonique, Jehan Soudan et Paul Bonnetain furent ses amants. Arthur Meyer, lui, doit sa réputation de secrétaire de Blanche d'Antigny à sa très grande proximité avec celle-ci au début des années 1860 , 
tandis que Jean Lorrain doit celle de porte-plume de Liane de Pougy à l'amitié indéfectible qui les lie.

La plupart des autobiographies de courtisanes sont de véritables best-sellers alors même que le marché éditorial connait une saturation certaine vis-à-vis des physiologies et des romans de mœurs parisiennes qui mettent en scène le demi-monde. La description de cet univers constitue le fonds de commerce d'un certain nombre de journalistes et d'hommes de lettres, et compte tenu du fonctionnement du marché de l'édition à cette époque (Mollier 1988), prêter sa plume à une courtisane peut être plus rentable que de publier un ouvrage en son nom; surtout lorsqu'on est encore un écrivain inconnu. Lorsqu'il écrit les Mémoires de Rigolboche, Ernest Blum est " poussé par les besoins d'un début laborieux et ayant de nombreuses charges ", l'opération lui rapporte environ 20000 francs, une somme plus que considérable pour l'époque ${ }^{47}$. Ce contexte explique la proximité stylistique entre les mémoires de courtisanes et la littérature panoramique galante publiés sous la Monarchie de Juillet et le Second Empire. Louis Huart, par exemple, l'un des deux co-auteurs des Mémoires de Rigolboche est journaliste et feuilletoniste, codirecteur de l'odéon et fondateur du théâtre des Folies nouvelles, auteur d'une Physiologie de la grisette illustrée par Gavarni et d'un Paris au bal ${ }^{48}$. Les Mémoires de Thérésa accumulent les stylèmes du Petit Journal et du Figaro ce qui est logique puisque que ses auteurs intercalent certains de leurs articles déjà parus à des chapitres inédits (Therenty 2013). Le travail de prêt-plume est alors très fréquent et il est ainsi piquant de voir Octave Mirbeau attaquer Paul Bonnetain comme étant le véritable auteur du Sarah Barnum ${ }^{49}$ publié par Marie Colombier alors que lui-même, à l'époque, est un prête-plume qui publie sous divers pseudonymes (Mirbeau 2004). Alors qu'Alexandre Dumas est célèbre pour avoir eu recours à de nombreux prêtes-plumes (Fillaire 2010), il aide Céleste Mogador à remanier son roman Les Voleurs d'or en drame de façon totalement désintéressée. Geste probable d'amitié pour son ancienne maitresse, devenue amie et marraine de sa fille Micaëlla Cordier, et qui fit brièvement la fortune de celle-ci.

Les collaborations littéraires qui se nouent entre courtisanes et hommes de lettres ont pour but principal de gagner de l'argent mais elles ne sont cependant pas d'ordre strictement professionnel. La plupart s'insèrent en réalité dans des liens amoureux et/ ou d'amitié bien plus vastes. Dans la seconde moitié du XIX ${ }^{e}$ siècle, les hommes de lettres du Tout-Paris médiatique ont la réputation d'être les "amants de cœur» des demi-mondaines, c'est-à-dire qu'ils ne payent pas leurs faveurs mais offrent à leurs belles des rôles au théatre, des articles ou encore des critiques dramatiques de complaisance. Dans le même temps, les salons tenus par les demi-mondaines représentent notamment un espace élitaire plus accessible aux hommes de lettres et aux artistes (encore inconnus) que les salons de la "bonne société ", voire un réseau directement concurrentiel de ces derniers (Gonzalez-Quijano 2017). Au point que l'on ne sait plus vraiment qui lance qui, et qui profite de qui. Léonide Leblanc, dans sa préface du livre Femmes de théâtre d'Alphonse Lemonnier évoque ainsi un certain Alphonse, journaliste, qui obtient les faveurs des débutantes en les citant dans ses critiques dramatiques et en leur écrivant des lettres de recommandation pour différents théâtres ${ }^{50}$. Tandis qu'Arthur Meyer, dans ses Mémoires parus au début du $\mathrm{XX}^{\mathrm{e}}$ siècle, se demande "qui se souvient encore, à ce propos, de la belle Mlle Delval, illustrée par le crayon de Marcelin, qui venait de fonder La Vie parisienne, un peu pour célébrer sa beauté51 ». La collaboration littéraire entre courtisanes et hommes de lettres 
s'intègre donc dans un ensemble d'associations et d'alliances beaucoup plus large et complexe, révélatrice de l'imbrication profonde entre demi-monde, milieu du théâtre et du journalisme. Mais surtout, elle implique de renoncer à une vision caricaturale des rapports entre "auteur.e déclarée" et "véritable auteur", à l'idée d'hommes exploitant le nom d'une femme illustre ou, au contraire, à celle de femmes abusant de secrétaires.

\section{Un seul « je » vénal ?}

Les relations entre hommes de lettres et demi-mondaines sont récurrentes, étroites et pétries d'ambiguïté. Ce sont aussi des relations qui ne sont pas fondées uniquement sur l'argent mais où chacun utilise à son profit la situation sociale de l'autre. La dédicace des Mémoires de Rigolboche au journaliste Manè [Henri de Pène] est d'ailleurs révélatrice de cette commune dépendance :

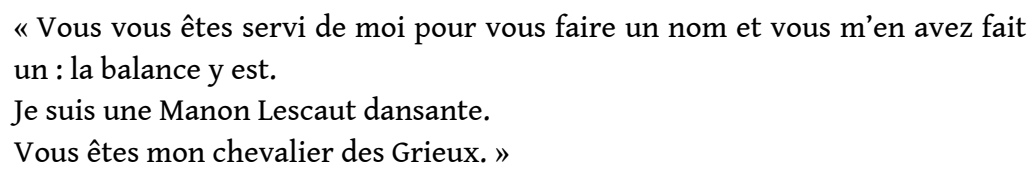

Cet étaiement réciproque est d'autant plus important qu'hommes de lettres et demimondaines partagent une position commune, celle de "parvenus ", au sein du demimonde et plus largement des élites parisiennes. Beaucoup d'hommes de lettres du demi-monde du Second Empire sont en effet d'anciens bohèmes de la Monarchie de Juillet à l'instar d'Arsène Houssaye ou de jeunes Rastignac comme Aurélien Scholl. Si l'affaire Dreyfus signe la naissance des intellectuels (Charle 1990), la professionnalisation des gens de lettres au XIXe siècle génère un véritable "prolétariat des lettres» exploité par les libraires et les éditeurs afin de vendre connaissance et distraction en attendant le succès. "La publication, cette prostitution de la pensée ", écrit Balzac dès 1828 dans son " avertissement » du Gars (Diaz 2013). Bien des écrivains $\mathrm{du}$ XIX ${ }^{\mathrm{e}}$ siècle se perçoivent comme vendant leur copie comme les filles publiques leur corps (Reverzy 2016) et dans ce contexte, leur collaboration à l'écriture d'une autobiographie de demi-mondaine ne peut que revêtir un sens tout particulier.

Se faire le prête-plume d'une courtisane c'est bien plus que vendre sa plume à une femme vénale, c'est devoir narrer sa vie et endosser son « je ». Et, à cet égard, il n'est pas impossible que le "contre-discours» de la femme vénale en tant que victime des hommes qui émerge des autobiographies de courtisanes (Koliopanos 2015, Sullivan 2016) soit autant la conséquence d'une écriture féminine de la prostitution que d'une identification des prêtes-plumes avec leurs auteur.es.

Les figures masculines négatives des mémoires de femmes galantes, en dehors des hommes liés à leur milieu d'origine (père absent, beau-père violent, etc.) sont souvent de jeunes hommes issus de la jeunesse dorée parisienne qui abandonnent lâchement leur maitresse en raison d'une maladie, d'une grossesse ou pour un mariage prometteur, c'est-à-dire des hommes avec lesquels les hommes de lettres sont en rivalité amoureuse et sociale directe. Durant le premier XIX siècle, le "Boulevard" représente un espace commun pour les hommes de la «bonne société » décidés à vivre en dandys et pour des jeunes gens partis de rien mais prêts à conquérir Paris à la pointe de la plume et du pinceau (Martin-Fugier 1990). À partir de la Monarchie de Juillet mais surtout sous le Second Empire, les espaces du Boulevard se confondent de plus en plus 
avec ceux du demi-monde: la littérature, le journalisme mais également la fréquentation conjointe des établissements publics et surtout des mêmes femmes galantes rapprochent des hommes d'origine sociale différente mais ayant adopté le même genre d'existence (Kushner 2013, Gonzalez-Quijano 2017). Dans ce contexte, la fréquentation des demi-mondaines n'est pas seulement une réponse à la «misère sexuelle » des célibataires, à l'ennui ou au malthusianisme de la sexualité conjugale, mais également une pratique destinée à garantir, magnifier ou à consolider, masculinité et statut social. Les figures de "l'amant de cœur ", du " protecteur attitré " et du «souteneur » présentes dans les Mémoires de courtisanes et plus largement dans la littérature prostitutionnelle, partitionnent ainsi les hommes fréquentant les prostituées entre "ceux qui paient ", «ceux qui obtiennent gratuitement » et " ceux qui en tirent profit ». Mais alors que les hommes de lettres, à l'instar d'Alexandre Dumas fils avec Marie Duplessis, n'ont pas les moyens financiers d'être les protecteurs uniques et officiels d'une courtisane et doivent se contenter de la position d'amant de cœur, il est tentant de représenter les hommes qui peuvent acheter leurs faveurs sous un jour défavorable... Ce que fait d'ailleurs Alexandre Dumas fils dans La Dame aux Camélias si l'on compare les portraits d'Armand Duval et du vieux duc, inspiré par le richissime comte de Stackelberg, l'un des derniers protecteurs connus de Marie Duplessis.

Le positionnement vis-à-vis de La Dame aux Camélias et l'intertextualité entre les œuvres de Céleste Mogador et Valtesse de la Bigne repérés par Courtney Sullivan s'intègre en réalité dans un ensemble beaucoup plus vaste d'allusions et de sous-entendus quant au rôle des journalistes dans le fonctionnement du demi-monde. Aux pseudonymes par lesquels les amants de ces dames sont désignés, et qui font que la plupart des mémoires du corpus s'apparentent à des romans à clefs, s'ajoutent une foule de private jokes comprises des seuls initiés du demi-monde de la presse (Therenty 2013). Hommes de lettres et demi-mondaines sont tout deux soumis à la déesse réclame et à la nécessité de se vendre, ce qui impacte profondément l'écriture de la prostitution. La lecture des mémoires de courtisanes frappe ainsi par sa relative homogénéité tant au niveau de l'invraisemblance des carrières prostitutionnelles que du style et de sa proximité avec la petite presse et les romans de la vie parisienne. Donnant ainsi l'impression d'une supercherie dont courtisanes et hommes de lettres se font les complices au détriment du lecteur.

Les termes désignant l'autobiographie ne deviennent courants en Europe qu'à partir du milieu du XIX ${ }^{e}$ siècle et leur apparition correspond à une période d'essor du genre luimême. Mais les textes présentés ici se détachent de la tradition d'écriture de mémoires pratiquée par de nombreuses femmes de l'aristocratie et de la grande bourgeoisie pour s'inscrire davantage dans celle des "romans-mémoires de courtisane », de la littérature panoramique galante et des « romans de filles». Ce sous-genre autobiographique " qui ne choque ni les règles de la morale, ni la bienséance ${ }^{52}$ » est en effet le produit de collaborations entre demi-mondaines et hommes de lettres où le pacte de vérité entre l'auteur.e et lectorat est profondément malmené au profit de la rentabilité financière. Le portrait de la courtisane en tant que victime des hommes (d'argent) qui émerge est ainsi autant le reflet d'une écriture féminine de la prostitution que d'une identification des prêtes-plumes avec leurs auteur.es. Dans le contexte du XIX ${ }^{e}$ siècle où le livre devient un objet de consommation courante mais où de nombreuses voix s'élèvent pour dénoncer l'industrialisation de la littérature et la transformation du livre en marchandise (Chartier et Martin 1985, Thiesse 2000), l'autobiographie de courtisane 
apparait comme une mise en abyme de la vénalité où le récit de la prostitution devient lui-même un objet marchand et où la vénalité des auteures est redoublée par leur «prostitution littéraire ».

\section{BIBLIOGRAPHIE}

BENHAMOU, Noëlle. 1996. Filles, prostituées et courtisanes dans l'œuvre de Guy de Maupassant : représentation de l'amour vénal. Villeneuve d'Ascq : Presses universitaires du Septentrion.

BERLIÈRE, Jean-Marc. 1992. La Police des mœurs sous la IIIe République. Paris : Le Seuil.

BERNHEIMER, Charles. 1989. Figures of Ill Repute : Representing Prostitution in NineteenthCentury France. Durham, Cambridge Mass. : Harvard University Press.

BRAUND, Sydney. 1947. The "Courtisane" in the French Theatre from Hugo to Becque (1831-1885). Baltimore : John Hopkins Press.

CHABRILLAN, Céleste Vénard de. 2015. Courtesan and Countess : The Lost and Found Memoirs of the French Consul's Wife. Edited and translated by Jana Verhoeven, Alan Willey, and Jeanne Allen, Melbourne : Melbourne UP.

CHARLE, Christophe. 1990. Naissance des « intellectuels »(1880-1900). Paris : Éditions de Minuit. CHARTIER, Roger, Martin, Henri-Jean (dir.). 1985. Histoire de l'édition française, tome 3. Paris : Promodis.

CLAYSON, Hollis. 2003 [1991]. Painted Love : Prostitution in French Art of The impressionist Era. Los Angeles : J. Paul Getty Trust.

CORTEY, Mathilde. 2001. L'Invention de la courtisane au XVIIIe siècle dans les romans-mémoires des « Filles du monde» de Madame Meheust à Sade (1732-1797). Paris, Editions Arguments.

CORVISIER, Christian. 2007. Cléo de Mérode et la photographie : la première icône moderne. Paris : éditions du Patrimoine.

COSNIER, Colette. 2001. Le Silence des filles. De l'aiguille à la plume. Paris : Fayard.

COUTURIER, Maurice. 1995. La Figure de l'auteur. Paris : Seuil.

DIAZ, José-Luis. « Balzac, les courtisanes et les lupanars de la pensée », in Guillaume Pinson (dir.), Presse, prostitution, bas-fonds (1830-1930), en ligne : [http://www.medias19.org/index.php? id=13396], mis à jour le :13/06/2013.

DINET, Dominique. 2011. « L'éducation des filles de la fin du 18e siècle jusqu'en 1918 ». Revue des sciences religieuses, $\mathrm{n}^{\circ} 85,457-490$.

DOTTIN-ORSINI, Mireille \& GROJNOWSKI, Daniel. 2008. Un joli monde. Romans de la prostitution. Paris : Laffont.

DUMAS, Alexandre. 2006. Les Voleurs d'or : drame en cinq actes précédé d'un prologue d'après le roman de Céleste de Chabrillan ; édition critique présentée et établie par Réginald Hamel. Montréal : Stanké. 
EDELMAN, Bernard. 2004. Le Sacre de l'auteur. Paris : Le Seuil.

FILLAIRE, Bernard. 2010. Alexandre Dumas, Auguste Maquet et associés. Paris : Bartillat.

GIOLITTO, Pierre. 1983-1984. Histoire de l'enseignement primaire au XIXe siècle. Paris : Nathan.

GONZALEZ-QUIJANO, Lola. 2012. Filles publiques et femmes galantes. Des sexualités légitimes et illégitimes à l'intérieur des espaces sociaux et géographiques parisiens 1851-1914). Paris : EHESS, thèse de doctorat.

GONZALEZ-QUIJANO, Lola. 2015a. Capitale de l'amour. Filles et lieux de plaisirs à Paris au XIXe siècle. Paris : Vendémiaire.

GONZALEZ-QUIJANO, Lola. 2015b. « Raconter la prostitution : mémoires et autobiographies de femmes galantes du XIXe siècle », in Patrizia Caraffi (dir.), Écrire, dit-elle, Scrivere lei disse. Bologne : I libri di Emil, 97-110.

GONZALEZ-QUIJANO, Lola. 2017. «Salons, sociabilité et réseaux sociaux autour des grandes courtisanes du XIXe siècle » in Bernard Lachaise, Dominique Picco, Carole Carribon, Delphine Dussert-Galinat et Fanny Bugnon (dir.), Réseaux de femmes, femmes en réseaux (XVIeXXIe siècle). Bordeaux : Presses universitaires de Bordeaux, 305-316.

IUSO, Anna (dir.). 1999. Scritture di donne. Uno sguardo europeo. Sienne : Protagon editori toscani.

JOUANNY, Sylvie. 2002. L'Actrice et ses doubles : figures et représentations de la femme de spectacle à la fin du XIXe siècle. Genève : Droz.

KOLIOPANOS, Yagos. 2015. « Les prostitutions à travers le prisme de l'écriture de soi de la comtesse de Chabrillan à Louise Chardon » in Gabrielle Houbre, Isolde Pludermacher et Marie Robert (dir.), Prostitution. Des représentations aveuglantes. Paris : Musée d'Orsay/Flammarion, 103-111.

KUSHNER, Nina. 2013. Erotic Exchanges. The World of Elite Prostitution in Eighteenth-Century. Paris, Ithaca : Cornell University Press.

LAROCHELLE, Claudine (dir.). 2015. Je veux une maison faite de sorties de secours : réflexions sur la vie et l'œuvre de Nelly Arcan. Montréal : VLB éditeur.

LILTI, Antoine. 2005. Le Monde des salons : sociabilité et mondanité à Paris au XVIIIe siècle. Paris : Fayard.

LILTI, Antoine. 2014. Figures publiques : l'invention de la célébrité, 1750-1850. Paris : Fayard.

LORUSSO, Silvia. 2017. « La misogynie littéraire. Le cas Sand ». Revue italienne d'études françaises $n^{\circ}$, en ligne : [http://journals.openedition.org/rief/1473].

MARTIN-FUGIER, Anne. 1990. La Vie élégante ou la formation du Tout-Paris, 1815-1848. Paris : Fayard.

MARTIN-FUGIER, Anne. 2001. Comédienne : de Mlle Mars à Sarah Bernhardt. Paris : Le Seuil.

MATLOCK, Jann. 1994. Scenes of Seduction: Prostitution, Hysteria, and Reading Difference in Nineteenth-Century France. New York : Columbia University Press.

MAYEUR, Françoise. 1979. L'éducation des filles au XIXe siècle. Paris : Perrin.

MIRBEAU, Octave. 2004. Quand Mirbeau faisait le « nègre "... Cinq romans d'Octave Mirbeau publiés sous les pseudonymes d'Alain Bauquenne et de Forsan, textes présentés et annotés par Pierre Michel. Paris : Les Éditions du Boucher. 
MOLLIER, Jean-Yves. 1988. L'argent et les lettres. Histoire du capitalisme d'édition, 1880-1920. Paris : Fayard.

MOLLIER, Jean-Yves. 2006. «Les femmes auteurs et leurs éditeurs au XIXe siècle : un long combat pour la reconnaissance de leurs droits d'écrivains ». Revue historique, vol. 638, no. 2, 313-333.

MOSER, Françoise. 1935. Vie et aventures de Céleste Mogador. Paris : Albin Michel.

MOSSMANN, Carole A. 2009. Writing with vengeance: The Countess de Chabrillan's Rise from Prostitution. Toronto : University of Toronto Press.

PEETERS, Guy. 2017. Gaspard de Cherville, l'autre « nègre » d'Alexandre Dumas. Paris : Honoré Champion.

PLANTÉ, Christine. 1989. La petite sœur de Balzac. Essai sur la femme auteur. Paris : Le Seuil. REID, Martine (dir.). 2011. Les femmes dans la critique et l'histoire littéraire. Paris : Éditions Honoré Champion.

REVERZY, Eléonore. Portrait de l'artiste en fille de joie. La littérature publique. Paris : CNRS Éditions, 2016.

RICATTE, Robert. 1960. La Genèse de « la Fille Elisa ». Paris : Presses universitaires de France. ROGERS, Rebecca. 2007. Les Bourgeoises au pensionnat. L'éducation féminine au XIXe siècle. Rennes : Presses universitaires de Rennes.

SEYMOUR, Bruce. 1998. Lola Montez: a life. New York : Yale University Press.

STILLINGER, Jack. 1991. Multiple authorship and the myth of solitary genius. Oxford : Oxford University Press.

SULLIVAN, Courtney. 2016. The Evolution of the Courtesan Novel. From de Chabrillan to Colette. Palgrave Macmillan.

THERENTY, Marie-Eve, « Le récit de vie de vedette, l'invention d'un genre : Rigolboche, Thérésa, Paulus », Belphégor, n¹1, 2013, en ligne : [https://journals.openedition.org/belphegor/279].

THIESSE, Anne-Marie. 2000. Le Roman du quotidien. Lecteurs et lectures populaires à la Belle Epoque. Paris : Le Seuil.

VAN CRUGTEN-ANDRÉ, Valérie. 1997. Le Roman du libertinage, 1782-1815. Paris : H. Champion.

WAGNEUR, Jean-Didier \& Cesteur, Françoise (dir.). 2012. Les bohèmes, 1840-1870 : écrivains, journalistes, artistes. Seysalle : Champ Vallon.

\section{NOTES}

1. Ce corpus est composé d'ouvrages à caractère autobiographique en français dont les auteures déclarées sont des femmes ayant eu une activité prostitutionnelle entre la Monarchie de Juillet et la Première Guerre mondiale et qui ont été publiés entre 1847 pour les Mémoires de Lola Montès et 1977 pour Mes Cahiers bleus de Liane de Pougy.

2. «Femme savante, d'une pédanterie ridicule » selon le Trésor de la langue française.

3. Louis de Vallières [Edmond Alonnier et Joseph Décembre], Mémoires de Rose Pompon, Paris, Gennequin fils, 1877.

4. Edouard Eliçagaray, Emile-Marco de Saint-Hilaire, Mémoires d'une célèbre courtisane des environs du Palais-Royal, ou Vie et aventures de Mlle Pauline, surnommée la Veuve de la Grande armée, Paris, Terry, 1833. 
5. Anonyme, La Belle Cauchoise ou Mémoires d'une jolie normande devenue courtisane célèbre, Paris, Palais-Royal, 1822.

6. Staines Théodore, Les Oiseaux de nuit et les polkeuses des scènes publiques, Paris, J. Breauté, 1845.

7. Gustave Bourdin, Voyage autour de Pomaré, reine de Mabille, princesse du Ranelagh, grandduchesse de la Chaumière, par la grâce de la polka, du cancan et autres cachuchas, Paris, G. Havard, 1844

8. Louis Festeau, Les Hétaïres en 1860, Paris, Imprimerie Juteau, 1861.

9. Le Demi-monde, pièce crée au Théâtre du Gymnase le 20 mars 1855.

10. Défaut de prononciation qui consiste à lier les mots sans raison.

11. $\mathrm{F}^{\circ} 4$ « Ackinson Camille dite Paquita », BB1, APP.

12. Quillette, Mémoires d'une biche anglaise, Paris, A. Faure, 1864.

13. William Stephens Hayward, Skittles in Paris : a biography of a "fascinating woman", Londres, C. H. Clarke, 1864.

14. Ernest Blum \& Louis Huart, Mémoires de Rigolboche: ornés d'un portrait photographié par Petit et Trinquart, Paris, E. Dentu, 1860.

15. Thérésa [Ernest Blum, Henri Rochefort et Albert Wolff], Mémoires de Thérésa, écrits par ellemême, Paris, E. Dentu, 1865.

16. Lola Montez, Aventures de la célèbre danseuse racontées par elle-même, avec son portrait et un fac-similé de son écriture, Paris, chez tous les libraires, 1847.

17. Hugues de Cortal, Memoiren der Lola Montez, C. Schultz, Berlin, 1851.

18. Fantasio, 1er novembre 1932.

19. Eugénie Buffet, Ma vie, mes amours, mes aventures; confidences recueillies par Maurice Hamel, Paris, E. Figuières, 1930.

20. Henri Frémont, Mémoires de Casque d'or racontés par elle-même et publiés par Henri Frémont, Paris, Bibliothèque du Journal fin de siècle, 1902.

21. Caroline Otéro, Les Souvenirs et la vie intime de la Belle Otéro, Paris, Le Calame, 1926. Claude Valmont étant l'un des pseudonymes de Fernande Salzedo, romancière populaire et bourgeoise désargentée.

22. Albert Wolff, «Courrier de Paris », Le Figaro, 27 février 1886, p. 1.

23. Robert Ricatte, La Genèse de la « Fille Elisa », Paris, Presses Universitaires de France, 1960.

24. Célèbre courtisane du Second Empire, elle fut notamment la maitresse de Jacques Offenbach, d'Edouard Manet et du prince de Sagan. Elle fera l'éducation galante de Liane de Pougy et côtoiera les grandes courtisanes de la Belle Époque avant de se retirer du demi-monde en 1902.

25. Céleste Mogador, Mémoires de Céleste Mogador, Paris, Librairie Nouvelle, 1858.

26. Comme le montrent certaines de ces œuvres comme Yvette Guilbert, Chanteries du Moyenâge. Quarante chansons, recueillies et adaptées par Yvette Guilbert et harmonisées par Edmond Rickett, Paris, Heugel, 1926 ; Yvette Guilbert, Dix chansons du XVIIIe siècle, Paris Heuguel, 1929.

27. Auriant, "Paul Adam, "nègre" de Marie Colombier », Mercure de France, 1 aout 1933, p. 721-724.

28. Paul-Victor Stock, « Paul Bonnetain et Sarah Barnum », Mercure de France, 15 mars 1934, p. 666.

29. Liane de Pougy, Mes Cahiers bleus, Paris, Plon, 1977, p. 49.

30. Lola Montez, Anecdotes of Love Being a True Account of the Most Remarkable Events Connected with the History of Love, in All Ages and Among All Nations, New York, Dick \& Fitzgerald, 1858

31. Lola Montez, The Art of the Beauty, or, Secrets of a Lady's Toilet. With Hints to Gentlemen on the Art of Fascinating, by Madame Lola Montez, New York, Dick \& Fitzgerald, 1858.

32. Emilienne André, Secrets de beauté pour être belle : recueil de conseils utiles et pratiques pour les soins de la femme..., Paris, s.n, 1919. 
33. Lola Montez, Anecdotes of Love, op. cit., preface, p. I.

34. Rachile, « Les romans », Mercure de France, janvier 1900, p. 459.

35. Jules Clarétie, La Vie à Paris : 1880-1910, Paris, G. Charpentier et E. Fasquelle, 1881-1991, p. 233 (5 aout 1910).

36. Albert Wolff, « Courrier de Paris », art. cit.

37. Fanny Lear, Le Roman d'une Américaine en Russie, accompagné de lettres originales, Paris, A. Lacroix, 1875.

38. XXX, « Histoire d'un livre », Le Figaro, 8 octobre 1875.

39. Asmodée, « Mort de Fanny Lear », Gil Blas, 16 aout 1886.

40. Cora Pearl, op. cit., 1886, p. 2.

41. Albert Wolff, art. cit. Il évoque néanmoins le fait que Cora Pearl se serait montrée très accommodante sur le montant de ces indemnités.

42. Catalogue des mobilier, argenterie, objets d'art et tableaux garnissant l'hôtel de Mlle Cora Pearl... vente 24-25 mai 1877, s.n, 1877 ; Catalogue de tableaux modernes, pastels, aquarelles, dessins..., riches bijoux, argenterie, dentelles,... mobilier artistique..., objets d'art... le tout appartenant à madame Valtesse de La Bigne et garnissant son hôtel... vente... 2-7 juin 1902, Paris, impr. de la Gazette des beaux-arts, 1902.

43. Catalogue d'un bon mobilier, meubles en bois sculpté, tableaux et aquarelles, objets d'art et de curiosité..., le tout dépendant de la succession de Mlle E. Crouch dite "Cora Pearl"... vente... 30 septembre et... 1er octobre 1886, s.n, 1886.

44. « Tribunaux », Le Matin, 9 décembre 1886.

45. Auriant, art. cit., p. 721.

46. Marie Colombier, On en meurt, Paris, C. Marpon et Flammartion, 1886, p. III.

47. Albert Wolff, La Gloire à Paris, Victor Havard, 1886, p. 185.

48. Louis Huart, Physiologie de la grisette, Paris, Aubert, 1841 et Paris au bal, Paris, Aubert, 1845.

49. Octave Mirbeau, "Crime en librairie", Grimaces, 15 décembre 1883. L'affaire alla suffisamment loin pour qu'il y ait duel entre les deux hommes de lettres le 17 décembre 1883.

50. Alphonse Lemonnier, Les Femmes de théâtre, Paris, Achille Faure, 1865, pp. I-XXII. Le prénom de ce journaliste n'est pas choisi par hasard puisque le terme d'“alphonse" désigne à l'époque un homme entretenu par sa femme ou un souteneur.

51. Arthur Meyer, La Dame aux Violettes, Paris, Plon, 1912, p. 12.

52. Timothée Trimm, « Les Mémoires de Thérésa », Le Petit Journal, 18 février 1865.

\section{RÉSUMÉS}

La prostitution féminine semble avoir toujours été l'objet d'une abondante production textuelle et visuelle. Mais ce n'est qu'à partir de la seconde moitié du XIXe siècle que des prostituées prennent la plume pour s'exprimer et s'autoreprésenter. Leurs ouvrages, s'ils sont souvent des succès de librairie, suscitent de nombreuses polémiques et sont régulièrement attribués à des hommes de lettres. A partir d'un corpus de livres autobiographiques de courtisanes, cet article montre comment ce sous-genre littéraire, par la collaboration qu'il implique entre la courtisane et son porte-plume, brouille les frontières entre écriture masculine et féminine, autobiographie et roman, littérature et publicité. Le portrait de la courtisane en tant que victime des hommes 
(d'argent) qui en émerge est ainsi autant le reflet d'une écriture féminine de la prostitution que du sentiment de prostitution du prolétariat des lettres de cette époque.

Female prostitution seems to have always been the subject of an abundant textual and visual production. But it is only from the second half of the nineteenth century on, that female prostitutes start to write to express and represent themselves. Often successful, their books cause many controversies and are regularly attributed to men of letters. Based on a collection of autobiographies by courtesans, this article shows how this literary sub-genre, by its necessary collaboration between these women and men of letters, blurs the boundaries between masculine and feminine writing, autobiography and novel, literature and advertising. The portrait of the courtesan as a victim of (business)men is thus as much the reflection of female writing about prostitution, than that of a prostitution feeling among male writing proletariat at that time of press and edition development.

INDEX

Mots-clés : autobiographie, presse, écriture, genre, scandale, célébrité

Keywords : Autobiography, press, writing, gender, scandal, celebrity

\section{AUTEUR}

\section{LOLA GONZALEZ-QUIJANO}

Lola Gonzalez-Quijano est docteure en histoire contemporaine, elle a notamment publié Capitale de l'amour. Filles et lieux de plaisirs à Paris au XIXe siècle aux éditions Vendémiaire (2015). Après avoir étudié l'imbrication des rapports de classe et de sexe dans la construction des identités sexuelles à l'époque contemporaine, elle décide d'aborder le corps autrement et d'entamer des études d'ostéopathie. 